\title{
THE ASSOCIATION BETWEEN BULLYING, MALTREATMENT, AND PERSONALITY TRAITS AMONG ADOLESCENTS FROM ARMENIAN SCHOOLS IN LEBANON
}

\author{
Manoug Ibitian ${ }^{1}, \&$ Ahmad Oweini ${ }^{2}$ \\ ${ }^{1}$ Armenian Evangelical Peter and Elizabeth Torosian Junior School (Lebanon) \\ ${ }^{2}$ Department of Education, Lebanese American University (Lebanon)
}

\begin{abstract}
The aim of this study was to identify the association between bullying, child domestic maltreatment, and personality traits among adolescents within a group of Armenian schools in Lebanon. Participants were 185 students in grades 7 to 10, attending Armenian schools in Beirut and its suburbs. The study depended on the self-report method, where participants supplied demographic information and filled in three questionnaires: Illinois Bully Scale, International Child Abuse Screening Tool - Children's Version (ICAST - CH) and Personality Inventory for DSM-5 Brief Form (PID-5-BF) Child Age $11-17$. Results, calculated by using ANOVA and Pearson correlation, indicated a statistically significant difference at the level of physical abuse between "victim" and "control" groups. Also, there were significant correlations between the "antagonistic" and "disinhibition" maladaptive personality traits and being a bully. The outcome of this study indicated the necessity of promoting parental and school guidance for preventing physical abuse within school, as well as concentrating on identifying the bully personality implicated in antagonism and disinhibition maladaptive personality traits within adolescents attending Armenian schools in Lebanon. The study includes prevalence information on bullies and victims in these schools, gender differences among bullies and victims, and clinical and research implications.
\end{abstract}

Keywords: Bullying, Lebanon, Armenian, child maltreatment, personality traits.

\section{Introduction}

The negative consequences of maltreatment and bullying on children has been widely researched, and include such conditions as depression, posttraumatic stress disorder, health issues cognitive difficulties psychopathologic behaviors such as aggression, social problems, and externalizing behavior issues, and suicidal attempts (Björkqvist, Österman, \& Berg, 2011; Fahlberg \& Kershnar, 2003; Lopes, 2013; and Olweus, 2003).

More recently, there has been a shift in emphasis from researching the prevalence and emotional consequences of bullying to examining the personality traits of the bully and antecedents of the bullying personality traits, namely domestic maltreatment, and its effects on maladaptive personality traits, mostly aggression, borderline and antisocial personality (Shields \& Cicchetti, 2001).

Bullying is a universal phenomenon and is just as prevalent in Lebanon. Rabah (2006), and Zein (2001) identified alarming occurrences of bullying among students in private schools in Lebanon.

\section{Background of the study}

Family is the first place where children experience interpersonal relationships between their parents and siblings. In all families there is a member who is in a higher authoritative position allowing them to safeguard the well-being of those with less power (U.S. Department of Health \& Human Services, 2003). When the more powerful member (i.e. parent) uses his/her power through maltreatment of the weaker members of the family (i.e. the child) this relationship is identified as child domestic maltreatment or "child abuse"; whereas when it occurs between peers then it is considered as "bullying" (Olweus, 2003).

The significance of such early relationship schema in the child's life can be traced through a large number of studies that have focused on the high association between physical maltreatment, sexual victimization, and neglect at home with being a bully and a victim at school later (Björkqvist et al. 2011; Hong et al., 2012; Mohr, 2006; Shields \& Cicchetti, 2001). In other words, instead of using appropriate parental disciplinary techniques, child maltreatment is imposed, which, with time, is turned into the children's own violent behavior (i.e. bullying). This confirms the existence of an association between maltreatment and bullying (Hong et al. 2012; Lopes, 2013). On the other hand, a handful of studies correlate 
personality traits to the bully (USDHHS, 2003). These traits include: poor self-esteem, absence of impulse control, anxiety, depression, and tendencies of antisocial behavior (Ondersma et al., 2005; Putnam, 2003; Usta et al., 2008; \& Valen Morgan, 2012).

\section{The significance of the study}

The study is an important contribution to the field because it addresses CDM, bullying and personality traits on a minority ethnic group in Lebanon, namely, the Armenian adolescents. Armenians living in Lebanon are a minority ethnic group representing $4 \%$ of the total Lebanese population (Central Intelligence Agency, 2014).

The aim of the study is to help increase the predictability of identifying bullies in schools that are struck by serious bullying issues, and inform the practice of educators and helping professionals in both early identification and treatment of bullies.

\section{Hypotheses}

The authors propose the following hypotheses:

Hypothesis 1: Bullies and victims will not differ on physical abuse but they will differ from control group.

Hypothesis 2: Bullies and victims will not differ on sexual abuse but they will differ from control group.

Hypothesis 3: Bullies and victims will not differ on neglect but they will differ from control group. a bully.

Hypothesis 4: There is a positive correlation between the Antagonistic personality trait and being a bully.

Hypothesis 5: There is a positive correlation between the Disinhibition personality trait and being

\section{Methods}

This study is quantitative research that employs a one-way ANOVA and correlation analysis in order to test the hypotheses and interpret the data obtained. It uses a convenient sample that includes 185 Armenian students between the age of 12-17, from grades 7-10 attending one of the four targeted Armenian schools in Lebanon. The response rate of this study was $92 \%$. The gender distribution consisted of 79 males $(42.7 \%)$ and 106 females $(57.3 \%)$.

Family status distribution was as follows: father and mother together $=175(94.6 \%)$, father and mother separated $=5(2.7 \%)$, father deceased $=3(1.6 \%)$, and other $=2(1.1 \%)$.

Besides the consent form and a demographic sheet, the participants answered the Illinois Bully Scale (2001), the International Child Abuse Screening Tool-Children's Home Version (2009), and the Personality Inventory for DSM 5 Brief Form (2013).

\section{Results}

\subsection{Reliability testing}

The internal reliability of these scales was computed by calculating the Cronbach's alpha of each scale and subscale, all showing high reliability coefficients per the table below.

Table 1. Cronbach's alpha for the subscales used in the research.

\begin{tabular}{|c|c|c|}
\hline Scale & Previous Cronbach alpha & Current Cronbach alpha \\
\hline Bullying Perpetration & 0.87 & 0.779 \\
\hline Victimization & 0.88 & 0.773 \\
\hline Neglect & 0.83 & 0.709 \\
\hline Physical Abuse & 0.77 & 0.637 \\
\hline Sexual Abuse & 0.72 & 0.684 \\
\hline Antagonism & - & 0.535 \\
\hline Disinhibition & - & 0.659 \\
\hline
\end{tabular}

\subsection{Demographic and descriptive data for bullying and victimization}

Out of a total number of 185 students, $10.8 \%(n=20)$ were identified as bullies, $6.5 \%(n=12)$ as victims, $11.9 \%(\mathrm{n}=22)$ both who are bully and victim at the same time, $45.9 \%(\mathrm{n}=85)$ as control group who are neither bullies nor victims (uninvolved), and $24.9 \%(\mathrm{n}=46)$ as "borderline".

The cut off scoring of Bully and Victim subscales was used from prior research conducted by Juvonen et al. (2003), which was done as follows: Those students who fell 0.5 standard deviations above 
the sample mean of the Bully subscale and below the sample mean of the Victim subscale were identified as "bully"; students who fell 0.5 standard deviations above the sample mean of the Victim subscale and below the sample mean of the Bully subscale were identified as "victim"; students who fell 0.5 standard deviations above the sample mean on both Bully and Victim subscales were classified as "both"; those who fell below the sample mean on both Bully and Victim subscales were identified as "control"; Whereas, the rest of the sample was considered as "borderline".

\subsection{Hypothesis testing}

Hypothesis 1: Bullies and victims will not differ on physical abuse but they will differ from control group.

A one-way between-group ANOVA (Post Hoc, Benferroni) was conducted to explore the impact of physical abuse (punishment), sexual abuse, and neglect on bullying and victimization, as measured by Illinois Bully Scale (IBS). Subjects were divided into four groups according to their IBS score (Group 1: Bullies; Group 2: Victims; Group 3: Both; Group 4: Control).

There was a statistically significant difference at the $\mathrm{p}<.05$ level in physical abuse scores only for the victims $[\mathrm{F}(3,135)=8.9, \mathrm{p}=.00]$. Post Hoc comparisons using the Benferroni test indicated that the mean score of the Victim $(M=4.17, S D=2.72)$ was significantly different from Control $(M=1.74$, $\mathrm{SD}=2.44)$, whereas, Bully $(\mathrm{M}=1.80, \mathrm{SD}=2.12)$ did not differ significantly from Control. Thus, hypothesis 1 is partially accepted.

Hypothesis 2: Bullies and victims will not differ on sexual abuse but they will differ from control group.

There was not a statistically significant difference at the $[F(3,135)=4.4, p=.06] p<.05$ level in sexual abuse scores between the Bully and Victim. Post Hoc comparisons using the Benferroni test indicated that the mean score of the Control $(\mathrm{M}=.24, \mathrm{SD}=.75)$ was not significantly different from Bully and Victim. Thus, hypothesis 2 is rejected.

Hypothesis 3: Bullies and victims will not differ on neglect but they will differ from control group.

There was not a statistically significant difference at the $[F(3,135)=2.25, p=.085 p<.05$ level in neglect scores for the Bully and Victim. Post Hoc comparisons using the Benferroni test indicated that the mean score of the Control $(\mathrm{M}=2.86, \mathrm{SD}=3.30)$ was not significantly different from Bully and Victim. Thus, hypothesis 3 is rejected.

Hypothesis 4: There will be a positive correlation between the Antagonistic personality trait and being a bully.

To test the association between the variables of personality traits: antagonism and disinhibition, and bullying and victimization, Pearson's product-moment correlation coefficient analysis was conducted.

Hypothesis four was confirmed, as the results of the correlation indicated that Antagonistic personality trait is positively and significantly correlated with being bully $\mathrm{r}=.407, \mathrm{p}=.000$.

Hypothesis 5: There will be a positive correlation between the Disinhibition personality trait and being a bully.

Hypothesis five was confirmed, as the results of the correlation revealed that disinhibition personality trait was positively and significantly correlated with being bully $\mathrm{r}=.229, \mathrm{p}=.002$.

\section{Discussion and implications}

\subsection{Association between child domestic maltreatment, bullying and victimization}

Both results concur with other published studies such as Holt et al. (2007) research that concluded that both groups, the "both" (bully and victim) and the "victim", reported significantly higher levels of physical abuse. In fact, Holt et al.'s (2007) study was one of the rare studies which reported similar results, specifically to the variables of physical and sexual abuse (neglect was not included in their hypotheses). When both studies were compared, no clear similarity was drawn except in the following: a) the same questionnaire of bully/victim identification, that is, IBS was used, b) the same method of identifying the groups of "bully", "victim", "both", etc., was employed and, c) the sample used was also from an urban environment, i.e., adolescents living in an urban environment are usually expected to more exposed to violence and witness different kinds of aggression as compared to nonurban environments (environmental factor) (Holt et al., 2007). Further exploration is needed in order to identify the reason behind this similarity of results.

Additionally, this significant difference between the "victim" and the control group when it comes to physical abuse, is explained through the attachment theory where the connection between child maltreatment by an abusive parent or caregiver leads to the development of an insecure attachment (Koiv, 2012; Toth, Manly \& Cicchetti, 1992; Shields \& Cicchetti, 2001; Wolfe, Crooks, Chiodo, \& Jaffe, 2009), which consequently plays a negative role in establishing maladaptive relationships with peers at school (Koiv, 2012; Hong et al., 2012).

The absence of a significant difference between the "bully" and the control group could be explained through the social-ecological model of bullying and existence of a healthy bonding between the 
maltreated child and a caring non-abusive adult, like a teacher or a leader, who is able in buffering and preventing bullying incidents by encouraging a positive interacting environment for neutralizing the negative effect of the early experience that was established through insecure attachment to the parent or the caregiver (Hong et al., 2012). In fact, taking the family status of this study into consideration, where 94.6 $\%$ of the parents living together as a family and not divorced (low divorce rate), might be helpful in explaining the reason for not getting a significant result.

Another confounding variable could be the level of aggression broadcast on TV and the online social media, where the increase in the rate of violence broadcasting plays an additional role in elevating the level of aggression in children.

The "both" group (victims and bullies), however, which was not part of the predicted hypothesis of this study was found to be significantly different from the control group. This particular result concurs with other published studies where the bully/victim group reported significantly higher levels of sexual victimization (Holt et al, 2007).

As for the association between neglect and bullying, it was hypothesized that bullies and victims will not differ on neglect but they will differ from the control group. The results of this study did not find any significant difference between the bully and victim compared to the control group, which concurs with one study by Shields and Cicchetti (2001). In this case, too, the possible explanation for this outcome is again similar to the one given between the "bully" and the "control" regarding the physical abuse.

As for this research being conducted in a Lebanese Armenian culture, it did not add to the significance of the study. It seems that the main differences could be more explained through family and environmental factors that are not specific to the Armenian culture in particular.

\subsection{Relationship between antagonistic and disinhibition personality traits, and bullying}

In order to identify the bully, it was imperative to define the maladaptive personality traits that can characterize the bully. Thus, this study took into consideration two maladaptive personality traits, the antagonistic and the disinhibition personality trait and hypothesized a positive correlation between these two traits and being a bully. The results were in line with other studies that have indicated the presence of a positive relation between these two maladaptive traits and being a bully. In his study performed on 527 university students, Pontzer (2010) found a positive correlation between impulsive (disinhibition) trait and those who were categorized as a bully.

\section{Clinical implications of the study}

In this research the interest was on three levels: Parental level that is taken into consideration through CDM (home environment/nurture), School level through bullying (social environment/nurture), and Personal level through personality traits (nature). Thus, this study tackled these three vital levels that play a significant role in the life of the student and help the psychologists, school counselors, social workers, educators, and practitioners in creating successful prevention programs and curricula that take into consideration the interplay of these three levels. Furthermore, identifying the significant effect of physical punishment on victims and bully/victims of bullying, in addition to the significant effect of sexual abuse on bully/victims, can help clinicians, school counselors, teachers, etc. in investigating the occurrence of domestic physical abuse in victims of the bullying on one hand, and the occurrence of domestic physical and sexual abuse in bully/victims on the other hand.

\section{Limitations and future research}

A main limitation of this study is related to its generalizability beyond students in Armenian Schools in Lebanon. Future research could target more representative samples of the Armenian community in Lebanon in order to be able to generalize the results to the whole population, and to replicate the study within other communities within Lebanese society, including Armenians who are attending non-Armenian schools in Lebanon.

\section{References}

Björkqvist, K., Österman, K., \& Berg, P. (2011). Higher rates of victimization to physical abuse by adults found among victims of school bullying. Psychological Reports. 109 (1), 167-168. DOI: 10.2466/02.07.11.16.PR0.109.4.167-168.

Effects of Bullying. (n. d.). Retrieved April 20, 2014, from http://www.stopbullying.gov/at-risk/effects/ Fahlberg, V. and Kershnar, S. "Child Sexual Abuse Across Cultures: What We Know So Far." Commissioned by UNICEF. February 10, 2003, pp.1-40. 
Friedman, M. S., Marshal, M. P., Guadamuz, T. E., Wei, C., Wong, C. F., Saewyc, E. M., \& Stall, R. (2011). A Meta-Analysis of Disparities in Childhood Sexual Abuse, Parental Physical Abuse, and Peer Victimization Among Sexual Minority and Sexual Nonminority Individuals. American Journal of Public Health. 101(8), 1481-1494. DOI: 10.2105/AJPH.2009190009.

Gershoff, E. (2002) Corporal punishment by parents and associated child behaviors and experiences: a meta-analytic and theoretical review. Psychological Bulletin, 128 (4), 539-579.

GlasØ, L., Matthiesen, S. B., Birkeland Nielsen, M., \& Einarsen, S. (2007). Do targets of workplace bullying portray a general victim personality profile? Scandinavian Journal of Psychology, 48, 313-319. DOI: 10.1111/j.1467-9450.2007.00554.x

Haj-Yahia, M.M. and Tamish, S. (2001). The Rates of Child Sexual Abuse and Its Psychological Consequences as Revealed by a Study among Palestinian University Students. Child Abuse and Neglect, 25(10), 1303-1327. DOI:10.1016/S0145-2134(01)00277-0

Harris, S, \& Petrie, G. (2003). Bullying. The bullies, the victims, the bystanders. Lanham, MD: Scarecrow Press, Inc.

Holt, M. K., Finkelhor, D., Kantor, G. K. (2007). Hidden Forms of Victimization in Elementary Students Involved in Bullying. School Psychology Review, 36 (3), 345-360.

Hong, J., Espelage, D., Grogan-Kaylor, A. \& Allen-Meares, P. (2012).. Identifying potential mediators and moderators of the association between child maltreatment and bullying perpetration and victimization in school. Educational Psychology Review. 24(2), 167-186. DOI: 10.1007/s10648011-9185-4.

Juvonen, J., Graham, S., \& Schuster, M. A. (2003). Bullying Among Young Adolescents: The Strong, the Weak, and the Troubled. Pediatrics, 112(6), 1231-1237. DOI: 10.1542/peds.112.6.1231Koiv, K. (2012). Attachment Styles among Bullies, Victims and Uninvolved Adolescents. Online Submission.

Lopes, B.C. (2013). Differences Between Victims of Bullying and Nonvictims on Levels of Paranoid Ideation and Persecutory Symptoms, the Presence of Aggressive Traits, the Display of Social Anxiety and the Recall of Childhood Abuse Experiences in a Portuguese Mixed Clinical Sample. Clinical Psychology \& Psychotherapy, 20 (3), 254-266. DOI: 10.1002/cpp.800.

Mohr, A. (2006). Family variables associated with peer victimization: Does family violence enhance the probability of being victimized by peers? Swiss Journal of Psychology, 65(2), 107-116. DOI: $10.1024 / 1421-0185.65 .2 .107$

Olweus, D. (2003). A profile of bullying at School. Educational Leadership, 60(6), 12-17.

Ondersma, S. J., Chaffin, M. J., Mullins, S. M., \& LeBreton, J. M. (2005). A brief form of the Child Abuse Potential Inventory: development and validation. Journal of Clinical Child and Adolescent Psychology, 34(2), 301-311.

Pontzer, D. (2010). A Theoretical Test of Bullying Behavior: Parenting, Personality, and the Bully/Victim Relationship. Journal of Family Violence, 25(3), 259-273. DOI:

10.1007/s10896-009-9289-5

Putnam, F. W. (2003). Ten-year research update review: child sexual abuse. Journal of the American Academy of Child \& Adolescent Psychiatry, 42(3), 269-278

Rabah, J. Y. (2006). Bullying problems in Lebanese private schools: teachers' and administrators' perceptions (master's thesis). American University of Beirut, Lebanon.

Shields, A. \& Cicchetti, D. (2001). Parental maltreatment and emotion dysregulation as risk factors for bullying and victimization in middle childhood. Journal of Clinical Child Psychology, 30(3), 349-363.

Tani, F., Greenman, P. S., Schneider, B. H., \& Fregoso, M. (2003). Bullying and the Big Five: A Study of Childhood Personality and Participant Roles in Bullying Incidents. School Psychology International, 24(2), 131-46.

Toth, S. L., Manly, J. T., \& Cicchetti, D. (1992). Child maltreatment and vulnerability to depression. Development and Psychopathology, 49(1), DOI: http://dx.doi.org/10.1017/S0954579400005587

U.S. Department of Health \& Human Services. (2003). A coordinated response to child abuse and neglect: The foundation for practice. In Child Abuse and Neglect User Manual. Retrieved April 20, 2014, from https://www.childwelfare.gov/pubs/usermanuals/foundation/foundation.pdf

Wolfe, D. A., Crooks, C. C., Chiodo, D., \& Jaffe, P. (2009). Child Maltreatment, Bullying, Gender-Based Harassment, and Adolescent Dating Violence: Making the Connections. Psychology of Women Quarterly, 33 (1), 21-24. doi: 10.1111/j.1471-6402.2008.01469.x

Zein, I. B. (2001). A survey of the extent of bully/victim problems in private schools in Beirut (unpublished master's thesis). American University of Beirut, Lebanon. 